\title{
Ethnicity and acculturation: do they predict weight status in a longitudinal study among Asian, Hispanic, and non-Hispanic White early adolescent females?
}

This article was published in the following Dove Press journal:

Adolescent Health, Medicine and Therapeutics

12 January 2015

Number of times this article has been viewed

\section{Marie K Fialkowski' \\ Reynolette Ettienne ${ }^{2}$ \\ Yurii B Shvetsov ${ }^{3}$ \\ Rebecca L Rivera' \\ Marta D Van Loan ${ }^{4}$ \\ Dennis A Savaiano' \\ Carol J Boushey'}

'Department of Nutrition Science, Purdue University, West Lafayette, IN, USA; ${ }^{2}$ Department of Health and Kinesiology, Texas A\&M University, College Station, TX, USA; ${ }^{3}$ Epidemiology Program, University of Hawai' 'i Cancer Center, Honolulu, HI, USA; ${ }^{4}$ United States Department of Agriculture, ARS Western Human Nutrition Research Center, Obesity and Metabolism Unit, Davis, CA, USA
Correspondence: Marie K Fialkowski Department of Human Nutrition, Food, and Animal Sciences, University of Hawai'i at Mānoa, 1955 East West Rd, AgSci 216, Honolulu, HI 96822, USA Tel + I 8089568337

Fax + I 8089564024

Email mariekf@hawaii.edu
Background: The prevalence of overweight and obesity among adolescents has increased over the past decade. Prevalence rates are disparate among certain racial and ethnic groups. This study sought to longitudinally examine the relationship between overweight status ( $\geq 85$ th percentile according to the Centers for Disease Control and Prevention growth charts) and ethnic group, as well as acculturation (generation and language spoken in the home) in a sample of adolescent females.

Methods: Asian ( $n=160)$, Hispanic ( $n=217)$, and non-Hispanic White ( $n=304)$ early adolescent girls participating in the multistate calcium intervention study with complete information on weight, ethnicity, and acculturation were included. Multiple methods of assessing longitudinal relationships (binary logistic regression model, linear regression model, Cox proportionalhazards regression analysis, and Kaplan-Meier survival analysis) were used to examine the relationship.

Results: The total proportion of girls overweight at baseline was $36 \%$. When examining by ethnic group, the proportion varied with Hispanic girls having the highest percentage (46\%) in comparison to their Asian (23\%) and Non-Hispanic White (35\%) counterparts. Although the total proportion of overweight was $36 \%$ at 18 months, the variation across the ethnic groups remained with the proportion of Hispanic girls becoming overweight (55\%) being greater than their Asian (18\%) and non-Hispanic White (34\%) counterparts. However, regardless of the statistical approach used, there were no significant associations between overweight status and acculturation over time.

Conclusion: These unexpected results warrant further exploration into factors associated with overweight, especially among Hispanic girls, and further investigation of acculturation's role is warranted. Identifying these risk factors will be important for developing targeted obesity prevention initiatives.

Keywords: acculturation, adolescents, overweight, obesity, ethnic group

\section{Introduction}

Nationally representative data from 2009 to 2010 showed that among adolescent girls 12-19 years of age, 33\% were overweight ( $\geq 85$ th percentile according to Centers for Disease Control and Prevention (CDC) growth charts) and 17\% were obese ( $\geq 95$ th percentile). ${ }^{1}$ Non-Hispanic Black (45\%), Mexican American (40\%), and Hispanic (including Mexican Americans) (42\%) adolescents were more likely to be overweight than non-Hispanic White (28\%) adolescents. ${ }^{1}$ Results from the National Longitudinal Study of Adolescent Health (Add Health) also show the same disparities between 
race/ethnic groups. ${ }^{2}$ However, due to the lack of oversampling in national surveys for Asians, it is difficult to provide overweight and obesity prevalence for this specific ethnic group. Furthermore, the causes of obesity among Asian Americans have not been as extensively studied in the extant literature as compared to other ethnic groups. The increasing prevalence of overweight among youth is of great concern and may be linked to an increase in type 2 diabetes mellitus ${ }^{3}$ as well as hypertension. ${ }^{4}$

Hispanic and Asian population groups are steadily increasing in the United States. ${ }^{5,6}$ These two groups not only represent the largest proportion of foreign-born individuals in the US, ${ }^{6}$ but may also be the most diverse. The health of these populations can dramatically influence the health of the emergent US population as "currently one out of five children is an immigrant or a child of an immigrant". ${ }^{7}$ Flores and Brotanek described the "Healthy Immigrant Effect" indicating that these "new" populations seem to have dramatically lower risks for developing certain diseases and health conditions. ${ }^{8}$ Surprisingly, immigrant adolescents and adults who were less acculturated to the American way of life were healthier regardless of socioeconomic status or lack of health insurance. ${ }^{8}$

However, researchers have shown that this protective effect seems to decrease with acculturation to the new country of origin. ${ }^{8,9}$ Acculturation, in brief, signifies the social, psychological, and behavioral changes consequent to immigration. ${ }^{10}$ Hazuda et al $^{11}$ defined acculturation as a multidimensional process in which individuals whose primary learning has been in one culture (eg, Mexican culture) take over characteristic ways of living from another culture (eg, Western culture). Acculturation as such encompasses any and all changes that occur following (prolonged) "contact" between individuals and groups of differing cultural backgrounds. ${ }^{12}$ Longer residency in the US has been associated with poorer health outcomes and this is especially evident when comparing first with later generations. ${ }^{13}$ Changes in dietary habits, physical activity, and health behaviors to closely mimic that of the host country have all been identified as critical factors. ${ }^{14-16}$ Previous studies have examined acculturation (primarily generation) and overweight status in children. ${ }^{2,13}$ However, both of these studies used data from the Add Health study, which represented a sample from almost 2 decades ago, and used a cross-sectional analysis.

The present study examined the development of overweight and obesity among early adolescent Asian, Hispanic, and nonHispanic White girls followed over 18 months, and whether these changes were influenced by acculturation. During the period of adolescence, dietary behaviors, changes in physical activity, as well as changes in body composition (such as location and quantity of body fat) typically occur, making this a critical age/stage to examine the influence of acculturation. ${ }^{17}$ The hypotheses for this study were 1) greater development of overweight/obesity would occur among the Hispanic girls followed by the non-Hispanic White and Asian girls and 2) less acculturated Asian and Hispanic adolescent girls would have a lower risk of becoming overweight/obese compared to those who were more acculturated.

\section{Methods}

This was a secondary analysis of the participants from the Adequate Calcium Today project, a multistate, schoolrandomized educational intervention (Arizona, California, Hawai' $i$, Indiana, Nevada, and Ohio) between the years of 2002 and 2005. The intervention was not related to changes in body weight. Recruitment methods have previously been described. ${ }^{18}$ Briefly, eligible participants in the study were sixth-grade girls who were at least $75 \%$ Asian, Hispanic, or non-Hispanic White based on self-reported race/ethnicity percentages of both biological parents. Sampling was limited in the Adequate Calcium Today project to Asians, Hispanics, and non-Hispanic Whites as this population was selected to examine another primary hypothesis (ie, at higher risk of osteoporosis later in life). ${ }^{18}$ However, this study population provided a unique opportunity to examine acculturation in an ethnically diverse sample; which included Asians as well as other female adolescents from geographic locations typically not sampled (Hawai'i) in the National Health and Nutrition Examination Survey (NHANES).

The methods for conducting anthropometric measurements have been previously described. ${ }^{18}$ In brief, each participant changed into lab-issued scrubs, removed their shoes, and then had both height and weight measured. Body mass index (BMI) was calculated using the formula weight $(\mathrm{kg}) /$ height $(\mathrm{m})^{2}$. The SAS program available from the National Center for Health Statistics of the CDC was used to determine the percentile rank for BMI-for-Age. ${ }^{19}$ Although childhood obesity is defined as at or above the 95 th percentile for age and sex, this study consolidated overweight and obese as a BMI at or above the 85 th percentile for age and sex according to the National Center for Health Statistics/CDC. ${ }^{19,20}$ Consolidation of these two BMI categories allowed for more inclusive analyses of the Asian participants, fewer numbers of whom were overweight and obese. Prior research on obesity in children and adolescents has also found that Asian American youths often do not make up sufficient sample sizes 
in epidemiological analyses. ${ }^{21}$ Physical activity from recreation for the previous year and sexual maturation (including pubertal staging) were self-reported by the participants and have been previously described elsewhere. ${ }^{18}$ With the exception of acculturation, all study measures were repeated at 12 months \pm 2 months after baseline and at 18 months \pm 4 months after baseline. Age was calculated by subtracting the date of each participant's visit from her date of birth.

For this analysis, complete information on acculturation was required. Acculturation was measured using two acculturation indicators: generation and language use..$^{22}$ Generation was categorized as first generation (born outside the US), second generation (born in the US and at least one parent born outside the US), third generation (girls and parents born in the US, and at least one grandparent born outside the US), and fourth generation (girls, parents, and grandparents born in the US). Language use ranged from least acculturated to most acculturated: no English spoken in the home, other language more than English used in the home, English same as another language used in the home, English more than another language used in the home, and only English. The Institutional Review Board of each participating site approved the study protocol, and written informed assents and consents were obtained from the girls and their parents, respectively.

Descriptive analyses were completed for girls in all race/ ethnic groups. Quantitative characteristics and primary study exposures (eg, acculturation indicators) were compared between race/ethnic groups by one-way analysis of variance. For comparison of categorical variables by race/ethnic group, chi-square analyses were done. The longitudinal relationships between race/ethnic groups and the likelihood for overweight were examined using Kaplan-Meier survival analysis from 10 months (ie, 12 months \pm 2 months after baseline) to 22 months (ie, 18 months \pm 4 months after baseline). This was completed across all race/ethnic groups without acculturation indicator stratification and within each race/ethnic group after stratification by acculturation indicators using the $\log$-rank test. For the purpose of this study, once a participant becomes classified as overweight or obese (at or above the 85th percentile) at any of the three visits, she is excluded from further analysis.

The variation in acculturation status among the nonHispanic White girls was limited; therefore, acculturation analyses were limited to the Asian and Hispanic girls. The longitudinal relationships of acculturation, based on the combined acculturation indicators (generation and language use in the home), to the likelihood of overweight across the
Asian and Hispanic race/ethnic groups were examined using a binary logistic regression model, a Cox proportional-hazards regression analysis, and a linear regression model adjusting for age (year), menarche status (yes/no), Tanner score, physical activity (metabolic equivalents/hour/week), state of residence, and intervention status (ie, school randomized to receive the educational intervention related to calcium). Multiple methods were used so that the potential longitudinal relationship between acculturation and overweight could be thoroughly examined. The IBM Statistical Package for the Social Sciences 22.0 (New York, NY, USA) was used for analyses. Only the results from the Kaplan-Meier analysis and the binary logistic models are shown due to all analytical methods yielding similar results.

\section{Results}

There were significant differences in the race/ethnicity distribution by state, generation, and language use within the home. For state, this difference was consistent with sampling methods. Table 1 shows the quantitative and categorical characteristics of the sample by race/ethnic group. The Hispanic girls had the lowest overall retention rate (76\%) compared to the Asian girls (94\%) and the non-Hispanic White girls (92\%). The cross-sectional prevalence of overweight among the sample is shown in Table 2. The Hispanic girls had the largest proportion of overweight compared to the Asian and non-Hispanic White girls at all three time points.

The probability for not becoming overweight was significantly associated with race/ethnic group (Figure 1). Over time, the proportion of Hispanic girls becoming overweight was higher than that among Asian and Non-Hispanic White girls $(P<0.001)$. The probability of becoming overweight was not significantly associated with acculturation score at any of the time points for either Asian or Hispanic girls (Table 3).

\section{Discussion}

This study involved a sample of early adolescent Hispanic, non-Hispanic White, and Asian girls from multiple locations around the country. The multiple sites allowed a substantial number of Hispanic and Asian girls to participate with a variety of cultural exposures. Previous studies ${ }^{13}$ demonstrated a protective effect of low acculturation toward a lower BMI and normal weight status among boys and girls in the 7 th-12th grades. We expected to find similar results given the diverse sample of girls and salient indicators of acculturation in our study. Despite these strengths, we were not able to duplicate the previous results. We found no significant relationship 
Table I Quantitative and categorical characteristics among Asian $(n=160)$, Hispanic $(n=217)$, and non-Hispanic White ( $n=304)$ early adolescent girls completing acculturation questions

\begin{tabular}{|c|c|c|c|c|}
\hline & Asian & Hispanic & Non-Hispanic White & Total \\
\hline \multicolumn{5}{|l|}{ Mean \pm standard deviation ${ }^{a}$} \\
\hline \multicolumn{5}{|l|}{ Age (years) by measurement visit*** } \\
\hline 0 month & $11.7 \pm 0.4^{x}$ & $11.8 \pm 0.5^{x}$ & $12.0 \pm 0.6^{y}$ & $11.8 \pm 0.5$ \\
\hline 12 months & $12.6 \pm 0.4^{x}$ & $12.8 \pm 0.5^{y}$ & $13.0 \pm 0.6^{z}$ & $12.8 \pm 0.5$ \\
\hline 18 months & $13.1 \pm 0.4^{x}$ & $13.4 \pm 0.5^{y}$ & $13.5 \pm 0.6^{z}$ & $13.4 \pm 0.5$ \\
\hline \multicolumn{5}{|l|}{ n (\%) } \\
\hline \multicolumn{5}{|l|}{ Number of girls by measurement visit } \\
\hline 0 month & $160(24)$ & $217(32)$ & $304(45)$ & 681 \\
\hline 12 months & $149(24)$ & $190(30)$ & $292(46)$ & 631 \\
\hline 18 months & $150(25)$ & $164(28)$ & $279(47)$ & 593 \\
\hline \multicolumn{5}{|l|}{ Study site ${ }^{c}$} \\
\hline Arizona & $\mathrm{I}(\mathrm{I})$ & $12 \mid(84)$ & $22(15)$ & 144 \\
\hline California & $48(4 I)$ & $68(59)$ & $0(0)$ & 116 \\
\hline Hawai'i & $105(80)$ & $4(3)$ & $22(17)$ & 131 \\
\hline Indiana & $3(2)$ & $19(15)$ & $108(83)$ & 130 \\
\hline Nevada & $0(0)$ & $5(23)$ & $17(77)$ & 22 \\
\hline Ohio & $3(2)$ & $0(0)$ & $135(98)$ & 138 \\
\hline \multicolumn{5}{|l|}{ Generation*** } \\
\hline First generation & $19(23)$ & $57(70)$ & $6(7)$ & 82 \\
\hline Second generation & $61(34)$ & $102(57)$ & $15(8)$ & 178 \\
\hline Third generation & $30(32)$ & $28(30)$ & $36(38)$ & 94 \\
\hline Fourth generation & $50(15)$ & $30(9)$ & $247(76)$ & 327 \\
\hline \multicolumn{5}{|l|}{ Language spoken in home*** } \\
\hline No English & $27(38)$ & $42(58)$ & $3(4)$ & 72 \\
\hline Other language more than English & II (24) & $34(74)$ & I (2) & 46 \\
\hline English same as another language & $18(20)$ & $70(76)$ & $4(4)$ & 92 \\
\hline English more than another language & $27(27)$ & $44(44)$ & $29(29)$ & 100 \\
\hline English only & $77(2 \mathrm{I})$ & $27(7)$ & $267(72)$ & 371 \\
\hline
\end{tabular}

Notes: ${ }^{\vee}$ Values with different superscripts $(x, y, z)$ indicate significantly different means between ethnic groups; ${ }^{b}$ percent totals not adding to 100 due to rounding; ${ }^{c}$ recruitment goals for race/ethnic groups based on population distributions at each site; a priori recruitment varied between sites. $* * * P<0.000$ I, between race/ethnic groups.

between the acculturation variables and probability for overweight regardless of the analytical approach used.

However, we were able to demonstrate that these race/ ethnic groups possess significantly different overweight

Table 2 Overweight status of early adolescent girls at three time points ( $n=68 \mathrm{I}$ at baseline)

\begin{tabular}{|c|c|c|c|c|}
\hline & Asian & Hispanic & $\begin{array}{l}\text { Non-Hispanic } \\
\text { White }\end{array}$ & Total \\
\hline \multicolumn{5}{|c|}{ Overweight status by measurement visit ${ }^{\mathrm{b}, * * *} \mathbf{n}(\%)^{\mathrm{a}}$} \\
\hline \multicolumn{5}{|l|}{0 month } \\
\hline 0 (no) & $124(78)$ & $117(54)$ & $198(65)$ & $439(64)$ \\
\hline I (yes) & $36(23)$ & $100(46)$ & $106(35)$ & $242(36)$ \\
\hline \multicolumn{5}{|l|}{12 months } \\
\hline 0 (no) & $123(83)$ & $96(5 \mathrm{I})$ & $184(63)$ & $403(64)$ \\
\hline I (yes) & $26(17)$ & $93(49)$ & $108(37)$ & $227(36)$ \\
\hline \multicolumn{5}{|l|}{18 months } \\
\hline 0 (no) & $123(82)$ & $74(45)$ & $184(66)$ & $381(64)$ \\
\hline I (yes) & $27(18)$ & $89(55)$ & $95(34)$ & $211(36)$ \\
\hline
\end{tabular}

Notes: aPercentages do not add to 100 due to rounding; based on growth charts developed by $\mathrm{NCHS} / \mathrm{CDC}, 0=<85$ th percentile and $\mathrm{I}=\geq 85$ th percentile. $* * * P<0.000$ I, between race/ethnic groups.

Abbreviations: NCHS, National Center for Health Statistics; CDC, Centers for Disease Control and Prevention. profiles. We found that Hispanic adolescent girls had a significantly lower probability of not being overweight compared to Asian or non-Hispanic White adolescent girls. In addition, in this study Hispanic adolescent girls had a larger proportion of overweight/obesity than in national surveys. In 2007-2008 and 2009-2010 NHANES, prevalence rates of approximately $40 \%$ were reported ${ }^{1,21}$ compared to $46 \%-55 \%$ in this study. This may be due to the Hispanic sample being primarily Mexican American. Previously, Gordon-Larsen et $\mathrm{a}^{13}$ found that acculturation and overweight among Hispanic adolescent immigrants to the US from Puerto Rico, Cuba, and Mexico displayed different profiles. The prevalence for overweight increased as generation increased in all groups except for the adolescents of Mexican origin. Coinciding with these results, Popkin ${ }^{23}$ identified Mexico as having a higher prevalence of overweight among the entire population, with the prevalence being highest in Northern Mexico, compared to other Latin American countries. This suggests that Mexico's proximity to the US and the high traffic across the border may mean that this ethnic group is 


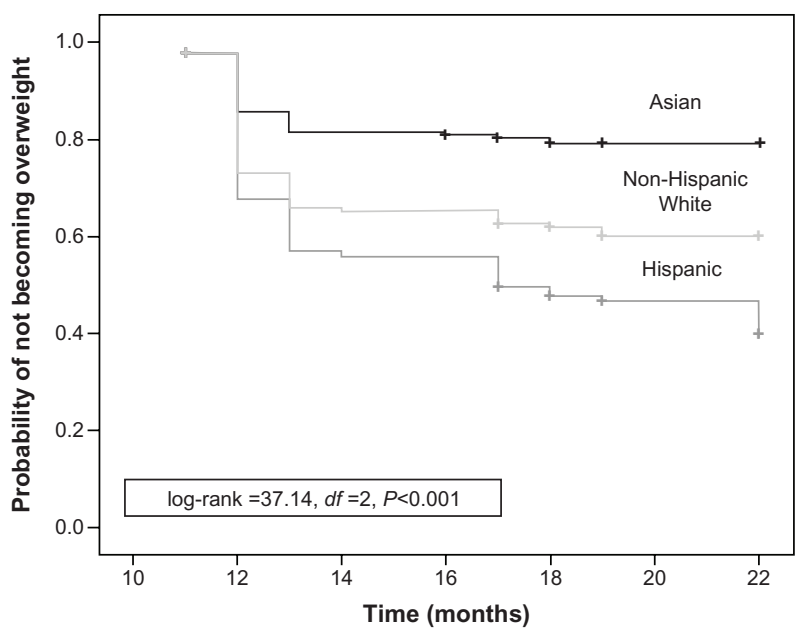

Figure I Kaplan-Meier survival curve showing probability for not becoming overweight among early adolescent girls.

Notes: Probability for not becoming overweight stratified by race/ethnic group from 10 months (post-baseline) to 22 months. Cumulative survival is the probability of not becoming overweight.

more acculturated to the US prior to immigration. This is a research question warranting further exploration. Maturation was also examined as a potential factor, because it has been shown to differ by ethnic group ${ }^{24}$ and menarche and it may influence overweight and obesity. ${ }^{25}$ These factors were not relevant predictors for this sample of adolescent girls. Genetic profiling may be another important predictor for overweight and obesity as has been demonstrated in a previous study. ${ }^{26}$

Table 3 Binary logistic regression model(s) of the relationship of acculturation score and overweight status among early adolescent Asian and Hispanic girls at three time points followed over 18 months

\begin{tabular}{|c|c|c|c|c|}
\hline \multirow[t]{2}{*}{ Time } & \multirow[t]{2}{*}{ Model $^{\mathrm{a}}$} & \multicolumn{3}{|c|}{ Acculturation score } \\
\hline & & OR & $(95 \% \mathrm{Cl})$ & $P$ \\
\hline \multicolumn{5}{|l|}{ Asian $(n=160)$} \\
\hline \multirow[t]{2}{*}{0 month } & 1 & 0.91 & $(0.66,1.25)$ & 0.57 \\
\hline & 2 & 0.98 & $(0.66,1.45)$ & 0.91 \\
\hline \multirow[t]{2}{*}{12 months } & 3 & 0.88 & $(0.6 \mathrm{I}, \mathrm{I} .27)$ & 0.49 \\
\hline & 4 & 0.88 & $(0.56,1.40)$ & 0.59 \\
\hline \multirow[t]{2}{*}{18 months } & 5 & 0.27 & $(0.07,1.12)$ & 0.07 \\
\hline & 6 & 0.29 & $(0.48,1.77)$ & 0.18 \\
\hline \multicolumn{5}{|c|}{ Hispanic $(n=2 \mid 7)$} \\
\hline \multirow[t]{2}{*}{0 month } & 1 & 1.08 & $(0.82,1.43)$ & 0.57 \\
\hline & 2 & 1.08 & $(0.81,1.45)$ & 0.59 \\
\hline \multirow[t]{2}{*}{12 months } & 3 & 1.05 & $(0.78, I .4 I)$ & 0.76 \\
\hline & 4 & 1.04 & $(0.77,1.43)$ & 0.79 \\
\hline \multirow[t]{2}{*}{18 months } & 5 & 0.56 & $(0.25,1.27)$ & 0.16 \\
\hline & 6 & 0.59 & $(0.25,1.39)$ & 0.23 \\
\hline
\end{tabular}

Notes: aodels I, 3, and 5 are unadjusted; Model 2 includes age, state of residence, menarche status, Tanner score, and physical activity; and Models 4 and 6 include these covariates and intervention status at 12 and 18 months.

Abbreviations: $\mathrm{OR}$, odds ratio; $\mathrm{Cl}$, confidence interval.
An interesting observation is that of the three race/ethnic groups, Hispanic girls had the lowest retention rates. Since this group was predominately Mexican American, frequent travel between the US and Mexico during the summer months has been shown to occur. Hispanic recruitment was the primary goal in California, Arizona, and Indiana, to increase the chance for Mexican American recruitment, which may have played a role in increased travel of the participants between the US and Mexico. If a majority of the girls lost to follow-up were lost due to this reason, it could have potentially impacted our results because of shorter follow-up of girls with lower acculturation. Despite this possibility, the girls lost to follow-up were found to be more acculturated (therefore, have a higher acculturation score) than those who completed follow-up (data not shown). Thus, the poor retention rates of Hispanic girls were unlikely to have impacted the acculturation analysis results.

Our results may differ from other studies for various reasons. Acculturation is a dynamic process and can be assessed in multiple ways. ${ }^{22}$ The brief approach of using language spoken in the home and generation status as a proxy for acculturation may have limited our ability to take advantage of the acculturation range, especially with regard to BMI. Montez and Eschbach ${ }^{27}$ found that these two acculturation indicators were significantly associated with unfavorable dietary profiles among non-pregnant women that would likely lead to overweight; however, measures of weight status were not examined in their cross-sectional study. Utilizing a multidimensional measure of acculturation status may be important to capture the breadth of acculturation. Other limitations of the present study include the use of secondary data for analysis, which may have contributed to a lack of power as well as the initial recruitment sample of girls not being based on acculturation or its effects on body weight, instead on bone development. The conflicting nature of these results implies a need for future research in this area. To further explore this phenomenon, a broader sample of boys and girls representing various race/ethnic groups and larger age ranges is recommended. Consistent with the study reported here, inclusion of multiple sites across the US is important, as regional locations within the US may modulate acculturation levels in individuals. Inclusion of socioeconomic and/or demographic factors in future studies may provide insight into their effects on acculturation and health, although a study by Gordon-Larsen et $\mathrm{al}^{28}$ revealed that the socioeconomic factors, income, and parental education level did not uniformly affect prevalence of overweight in adolescents. Another study among 
girls residing in California showed an association between socioeconomic status, acculturation, percentage body fat, and risk of overweight in Asian Americans but not Mexican Americans. ${ }^{29}$ In addition, information related to dietary patterns may be important to collect as diet is an important indicator of acculturation. ${ }^{30}$

\section{Conclusion}

NHANES data over the years have demonstrated the differences in overweight/obesity among select race/ethnic groups, which is consistent with our results. Unique to this study is the new information with regard to early adolescent Asian girls having lower rates of overweight/obesity than their Hispanic and non-Hispanic White counterparts. In addition, Asian girls had the lowest proportion of those becoming overweight/obese. The lack of an association between acculturation and BMI suggests that the effects of acculturation may vary and depend on the life stage. Sociocultural issues influencing these race/ethnic differences in overweight/obesity merit investigation, especially in light of the potential impact of adolescent obesity on the onset of diabetes and hypertension.

\section{Acknowledgments}

This study was funded by the Initiative for Future Agriculture and Food Systems; United States Department of Agriculture Cooperative State Research, Education, and Extension Service Grant 00-52102-9696; and Research Centers in Minority Institutions Award P20 RR011 091, National Center for Research Resources/NIH. At the time of the study, coauthor MKF was supported by Purdue University's Andrews Fellowship Program. Coauthor RE was supported by the National Cancer Institute, Nutritional and Behavioral Cancer Prevention in a Multiethnic Population postdoctoral fellowship (R25 CA 90956). We thank the participants and their parents for their willingness to participate in the study.

\section{Author contributions}

MKF and CJB made substantial contributions to conception and design; CJB, DAS, and MDVL were responsible for the acquisition of data; MKF, CJB, RE, YBS, and RLR contributed to analysis and interpretation of data; MKF drafted the article and led the writing team; CJB, RE, YBS, RLR, MDVL, and DAS revised the manuscript critically for important intellectual content; and all authors read and approved the final version and agreed to be accountable for all aspects of the work in ensuring that questions related to the accuracy or integrity of any part of the work are appropriately investigated and resolved.

\section{Disclosure}

The authors report no conflicts of interest in this work.

\section{References}

1. Ogden CL, Carroll MD, Kit BK, Flegal KM. Prevalence of obesity and trends in body mass index among US children and adolescents, 1999-2010. JAMA. 2012;307(5):483-490.

2. Popkin BM, Udry JR. Adolescent obesity increases significantly in second and third generation US immigrants: the National Longitudinal Study of Adolescent Health. J Nutr. 1998;128(4):701-706.

3. Fagot-Campagna A, Saadinem JB, Flegal KM, Beckles GL. Third National Health and Nutrition Examination Survey. Diabetes, impaired fasting glucose, and elevated $\mathrm{HbA1c}$ in US adolescents: the Third National Health and Nutrition Examination Survey. Diabetes Care. 2001;24(5):834-837.

4. Flynn JT, Falkner BE. Obesity hypertension in adolescents: epidemiology, evaluation, and management. J Clin Hypertens. 2011;13(5):323-331.

5. Pew Research Center. The Rise of Asian Americans; 2012. Available from: http://www.pewsocialtrends.org/2012/06/19/the-rise-ofasian-americans/. Accessed September 4, 2012.

6. Larsen L. The Foreign Born Population in the United States: 2003. Report No: Current Population Reports, P20-551. Washington, DC: US Census Bureau; 2004.

7. Nguyen HH. Acculturation in the United States. In: Sam DL, Berry JW, editors. The Cambridge Handbook of Acculturation Psychology. Cambridge, MA: Cambridge University Press; 2006:311-330.

8. Flores G, Brotanek J. The healthy immigrant effect: a greater understanding might help us improve the health of all children. Arch Pediatr Adolesc Med. 2005;159:295-297.

9. Abraído-Lanza AF, Chao MT, Flórez KR. Do healthy behaviors decline with greater acculturation? Implications for the Latino mortality paradox. Soc Sci Med. 2005;61(6):1243-1255.

10. Cabassa LJ. Measuring acculturation: where we are and where we need to go. Hisp J Behav Sci. 2003;25(2):127-146.

11. Hazuda HP, Haffner SM, Stern MP, Eifler CW. Effects of acculturation and socioeconomic status on obesity and diabetes in Mexican Americans. The San Antonio heart study. Am J Epidemiol. 1988;128(6):1289-1301.

12. Sam DL. Acculturation: conceptual background and core components. In: Sam DL, Berry JW, editors. The Cambridge Handbook of Acculturation Psychology. Cambridge: Cambridge University Press; 2006:11-26.

13. Gordon-Larsen P, Harris KM, Ward DS, Popkin BM. Acculturation and overweight-related behaviors among Hispanic immigrants to the US: the National Longitudinal Study of Adolescent Health. Soc Sci Med. 2003;57:2023-2034.

14. Unger JB, Reynolds K, Shakib S, Spruijt-Metz D, Sun P, Johnson CA. Acculturation, physical activity, and fast-food consumption among Asian-American and Hispanic adolescents. J Community Health. 2004;29(6):467-481.

15. Zimmerman EJ, Sodowsky GR. Influences of acculturation on Mexican American drinking practices: implications for counseling. J Multicult Couns Dev. 1993;21(1):22-35.

16. Marin G, Perez-Stable EJ, Marin BV. Cigarette smoking among San Francisco Hispanics: the role of acculturation and gender. Am J Public Health. 1989;79(2):196-198.

17. Alberga AS, Sigal RJ, Goldfield G, Prud'homme D, Kenny GP. Overweight and obese teenagers: why is adolescence a critical period? Pediatr Obes. 2012;7(4):261-273.

18. Weaver CM, McCabe LD, McCabe GP, et al; ACT Research Team. Bone mineral and predictors of bone mass in White, Hispanic, and Asian early pubertal girls. Calcif Tissue Int. 2007;81:352-363. 
19. Centers for Disease Control. A SAS Program for the CDC Growth Charts. Centers for Disease Control and Prevention; 2006. Available from: http://www.cdc.gov/nccdphp/dnpa/growthcharts/resources/sas. htm. Accessed March 7, 2007.

20. Kuczmarski RJ, Ogden CL, Guo SS, et al. 2000 CDC Growth Charts for the United States: Methods and Development. 11th ed. Washington: National Center for Health Statistics; 2002.

21. Ogden CL, Carroll MD, Curtin LR, Lamb MM, Flegal KM. Prevalence of high body mass index in US children and adolescents, 2007-2008. JAMA. 2010;303(3):242-249.

22. Cuellar I, Arnold B, Maldonado R. Acculturation rating scale for Mexican Americans-II: a revision of the original ARSMA scale. Hisp J Behav Sci. 1995;17:275-304.

23. Popkin BM. The nutrition transition and its health implications in lowerincome countries. Public Health Nutr. 1998;1(1):5-21.

24. Kaplowitz P. Pubertal development in girls: secular trends. Curr Opin Obstet Gynecol. 2006;18:487-491.

25. Biro FM, Greenspan LC, Galvez MP. Puberty in girls of the 21 st century. J Pediatr Adolesc Gynecol. 2012;25(5):289-294.
26. Garup N, Sandholt CH, Hansen T, Pederson O. Genetic susceptibility to type 2 diabetes and obesity: from genome-wide associate studies to rare variants and beyond. Diabetologia. 2014;57(8):1528-1541.

27. Montez JK, Eschbach K. Country of birth and language are uniquely associated with intakes of fat, fiber, and fruits and vegetables among Mexican-American women in the United States. J Am Diet Assoc. 2008;108:473-480.

28. Gordon-Larsen P, Adair LS, Popkin BM. The relationship of ethnicity, socioeconomic factors, and overweight in US adolescents. Obes Res 2003;11:121-129.

29. Schaefer SE, Salazar M, Bruhn C, Savaiano D, Boushey C, Van Loan MD. Influence of race, acculturation, and socioeconomic status on tendency toward overweight in Asian-American and Mexican-American early adolescent females. J Immigr Minor Health. 2009;11(3):188-197.

30. Unger JB, Reynolds K, Shakib S, Spruijt-Metz D, Sun P, Johnson CA. Acculturation, physical activity, and fast-food consumption among Asian-American and Hispanic adolescents. J Community Health. 2004;29:467-481
Adolescent Health, Medicine and Therapeutics

\section{Publish your work in this journal}

Adolescent Health, Medicine and Therapeutics is an international, peer-reviewed, open access journal focusing on health, pathology, and treatment issues specific to the adolescent age group. All aspects of health maintenance, preventative measures and disease treatmen interventions are addressed within the journal and practitioners from

\section{Dovepress}

all disciplines are invited to submit their work as well as healthcare researchers and patient support groups.. The manuscript management system is completely online and includes a very quick and fair peerreview system. Visit http://www.dovepress.com/testimonials.php to read real quotes from published authors.

Submit your manuscript here: http://www.dovepress.com/adolescent-health-medicine-and-therapeutics-journal 